\title{
Humedales turbosos de la Región de Aisén (Patagonia chilena): Clasificación y ordenación de la flora y la vegetación
}

\author{
Peat bog wetlands of Aisén Region (Chilean Patagonia): \\ Classification and ordination of flora and vegetation
}

\author{
Carlos Ramírez a*, Cristina San Martín b, Javier Amigo c, Miguel Álvarez d, \\ Osvaldo Vidal ${ }^{e}$, Yéssica Pérez ${ }^{b}$, Gisela Toledo ${ }^{f}$ \\ *Autor de correspondencia: a Pontificia Universidad Católica de Chile, Facultad de Ciencias Biológicas, \\ Departamento de Ecología, tel.: 56-63-2206227, Santiago, Chile, cramirez@uach.cl \\ ${ }^{\mathrm{b}}$ Universidad Austral de Chile, Facultad de Ciencias, Instituto de Ciencias de la Tierra y Herbario, Valdivia, Chile. \\ ${ }^{c}$ Universidad de Santiago de Compostela, Herbario, Facultad de Farmacia, España. \\ ${ }^{\mathrm{d}}$ Rheinische-Friedrich Wilhelms-Universität, INRES-Vegetationsökologie, Bonn, Alemania. \\ ${ }^{\text {e } U n i v e r s i d a d ~ d e ~ M a g a l l a n e s, ~ I n s t i t u t o ~ d e ~ l a ~ P a t a g o n i a, ~ P u n t a ~ A r e n a s, ~ C h i l e . ~}$ \\ ${ }_{\mathrm{f}}$ Corporación Nacional Forestal, Departamento de Vida Silvestre, Puerto Montt, Chile.
}

\begin{abstract}
SUMMARY
The peat bogs of Capitán Prat Province in Aisén Region in Chilean Patagonia were studied, raising 204 vegetation samples with plant sociological methodology, with which an initial table with 106 species of flora in its first column was built. With traditional methodology, this table was ordered using differential species and with multivariate classification and ordination statistics, all the possible ecological information contained in it was extracted. Four plant communities of the peat bog formation were determined: Gaultherio-Sphagnetum magellanici (Sphagnum peat bog), Schoeno andinus- Lepidothamnetum fonkii (ciprés enano peat bog), Cortaderio egmontianae-Schoenetum andinus (gramineous peat bog) and Drosero uniflorae-Donatietum fascicularis (pulvinate peat bog). The third Cortaderio egmontianae-Schoenetum andinus is proposed as a new plant association. The floristic similarity among them was high, even though they can be easily physiognomically differentiated. Multivariate ordination suggests that waterlogging and temperature, conditioned by relief and altitude, are important factors in the differentiation of peat bog communities. It is concluded that of these four associations, two -the Sphagnum peat bog and Lepidothamnus fonkii peat bog- correspond to the region of the evergreen Magellanic Forest, while the other two are northern outposts of the Magellanic Tundra Region, typical of the Southern Hemisphere, especially in Chile and New Zealand.
\end{abstract}

Key words: Peat bogs, plant associations, classification, ordination, Magellanic tundra.

\section{RESUMEN}

Se estudió la vegetación turbosa de la provincia de Capitán Prat en la Región de Aisén, Patagonia chilena, levantando 204 censos de vegetación con metodología fitosociológica, con ellos se construyó una tabla inicial con 106 especies vegetales en su primera columna. Con metodología tradicional se ordenó esta tabla utilizando especies diferenciales y con estadística multivariada de clasificación y ordenación, se extrajo toda la información ecológica encerrada en ella. Se determinaron cuatro asociaciones vegetales turbosas: Gaultherio-Sphagnetum magellanici (turbera esfagnosa), Schoeno andinus-Lepidothamnetum fonkii (turbera esfagnosa de ciprés enano), Cortaderio egmontianae-Schoenetum andinus (turbera graminosa) y Drosero uniflorae-Donatietum fascicularis (turbera pulvinada). La tercera, Cortaderio egmontianae-Schoenetum andinus se propone como una asociación vegetal nueva. La similitud florística entre ellas fue alta, aun cuando pueden diferenciarse fácilmente por su fisonomía. El análisis multivariado de ordenación, sugiere que la disponibilidad hídrica y la temperatura, condicionados por el relieve y la altitud, son factores importantes en la diferenciación de las comunidades. Se concluye que de estas cuatro asociaciones dos, las turberas esfagnosa y de ciprés enano, corresponden a la región de los bosques magallánicos perennifolios, mientras que las otras dos, son avanzadas septentrionales de la Región de la Tundra magallánica, propia del hemisferio Sur, especialmente de Chile y Nueva Zelanda.

Palabras clave: turberas, asociaciones vegetales, clasificación, ordenación, tundra magallánica. 


\section{INTRODUCCIÓN}

Aunque la flora vascular (especies) de la Región de Aisén (Chile) es conocida y se encuentra en el inventario de los recursos naturales de nuestro país (Álvarez et al. 2010, Ramírez et al. 2014a, San Martín et al. 2014a, Sandoval et al. 2016) no sucede lo mismo con las asociaciones que son aún poco estudiadas, como lo demuestra la escasa y reciente literatura al respecto (Álvarez et al. 2010, Ramírez et al. 2014a, Amigo et al. 2017). Si bien se han descrito grandes zonas de vegetación, aún falta por conocer las comunidades vegetales que conforman el paisaje original, ya que, por ejemplo, se suelen considerar muy importantes en el paisaje de la región las comunidades vegetales de praderas permanentes, formadas por flora alóctona que son secundarias y de origen antrópico y no originales del lugar (Ramírez et al. 2012). Sin embargo, existen muchas comunidades vegetales con alto grado de endemismos que aún son desconocidas, como las turberas investigadas en este trabajo.

Como en Chile, con excepción de la Región de Magallanes, el espesor de la capa de turba es relativamente reducida, las turberas son poco utilizadas para obtener combustible doméstico, pero en cambio están siendo explotadas para aprovechar los musgos del género Sphagnum, principalmente Sphagnum magellanicum (localmente "ponpon") exportado para horticultura, para cultivos en macetas y como absorbente en pañales de todo tipo (Vásquez 2008). Además, en algunas de estas turberas es frecuente encontrar rodales de Pilgerodendron uviferum (ciprés de Las Guaitecas) especie que produce apreciada madera de construcción, por su hermosa veta, alta resistencia a la humedad y especialmente, su fragancia. Lo comentado provoca problemas de conservación a las turberas.

Por todo lo anterior y teniendo en cuenta que el ser humano solo se interesa y protege aquello que conoce, el objetivo de este trabajo es estudiar los ecosistemas turbosos de la Región de Aisén, para dar a conocer su estructura florística y vegetacional y elaborar una clasificación fitosociológica actualizada de ellas, que permita diferenciarlas como unidades de paisaje (Rodríguez 2015). Para contribuir con este conocimiento a la explotación racional, junto a un manejo sustentable de ellas, se desea saber, ¿Cuántas especies vegetales componen la estructura de estas turberas? ¿Cuántas comunidades vegetales diferentes se presentan en ellas? y ¿Cuál de ellas muestra la mayor diversidad florística? La hipótesis es que los regímenes hídrico y térmico determinan la diferenciación y distribución altitudinal de las comunidades turbosas existentes en la región.

\section{MÉTODOS}

Lugar de trabajo. La Región de Aisén del General Carlos Ibáñez del Campo se ubica en el Sur de Chile entre los $43^{\circ}$ $38^{\prime}$ y $49^{\circ} 16^{\prime}$ de latitud Sur, entre el Océano Pacífico por el Oeste y la República Argentina por el Este. En la provincia
Capitán Prat de la Región de Aisén (Chile) se levantaron 204 censos de vegetación (relevé) con metodología fitosociológica en rodales considerados previamente y a simple vista, como pertenecientes a la formación vegetal turbera (Mueller-Dombois y Ellenberg 1974). La figura 1 muestra la zona trabajada y la ubicación de los censos de vegetación. Los relevamientos se realizaron en rodales fisonómica, florística y ecológicamente homogéneos, evitando zonas ecotonales hacia bosques u otra formación vegetal, de acuerdo a los requisitos de muestreo planteados por la fitosociología (Dengler et al. 2008). La nomenclatura sigue a Zuloaga et al. (2008) y Rodríguez y Marticorena (2019).

Procedimiento de terreno. La toma de datos en terreno se realizó entre los años 2007 y 2010 levantando censos de vegetación en parcelas homogéneas de $100 \mathrm{~m}^{2}(10 \mathrm{~m}$ x 10 $\mathrm{m})$ superiores al área mínima establecida para la vegetación de turberas (Knapp 1984). En cada censo se confeccionó una lista de las especies vegetales presentes y luego, se determinó la abundancia de los individuos de cada una, expresándola en porcentaje de cobertura de la parcela. Para coberturas inferiores a $1 \%$ se utilizaron los signos "+" (cruz) y "r" (erre), el primero cuando había varios individuos y el segundo cuando solo apareció un individuo de la especie en cuestión. Para cálculos posteriores, estos signos fueron subidos a la unidad, lo que aumenta la cobertura total de cada censo.

Análisis de datos. Los 204 censos de vegetación se reunieron en una tabla fitosociológica inicial, cuya primera columna lleva las especies y en ella se calculó la frecuencia, número de censos en que se presenta cada especie y la cobertura total de cada una sumando todas las coberturas donde está presente. Este valor se expresa en porcentaje de la parcela y puede sobrepasar varias veces el $100 \%$. Se calculó también un valor de importancia utilizando la frecuencia y la cobertura total de cada especie, expresada en porcentaje en relación a la suma de todas las especies de la tabla (Wikum y Shanholtzer 1978).

Las comunidades vegetales de la tabla fueron separadas utilizando especies diferenciales que se excluyen mutuamente en diferentes grupos de censos (Knapp 1984). Para cada una de ellas se confeccionó aparte una tabla parcial con las especies y el valor de importancia de ellas. La diferenciación y la nomenclatura utilizada sigue al Código de Nomenclatura Fitosociológica (Weber et al. 2000) y la clasificación sintaxonómica de las asociaciones vegetales fue tomada de Amigo et al. (2017).

Se construyó una tabla resumen ordenada de las cuatro comunidades vegetales identificadas utilizando el promedio de cobertura de cada especie, obtenido dividiendo la cobertura total por el número de censos de la tabla respectiva, subiendo o bajando los valores a la unidad cuando se presentaban decimales. Esta tabla fue utilizada para determinar las especies compartidas o exclusivas de una sola comunidad. También se calculó la similitud florística 


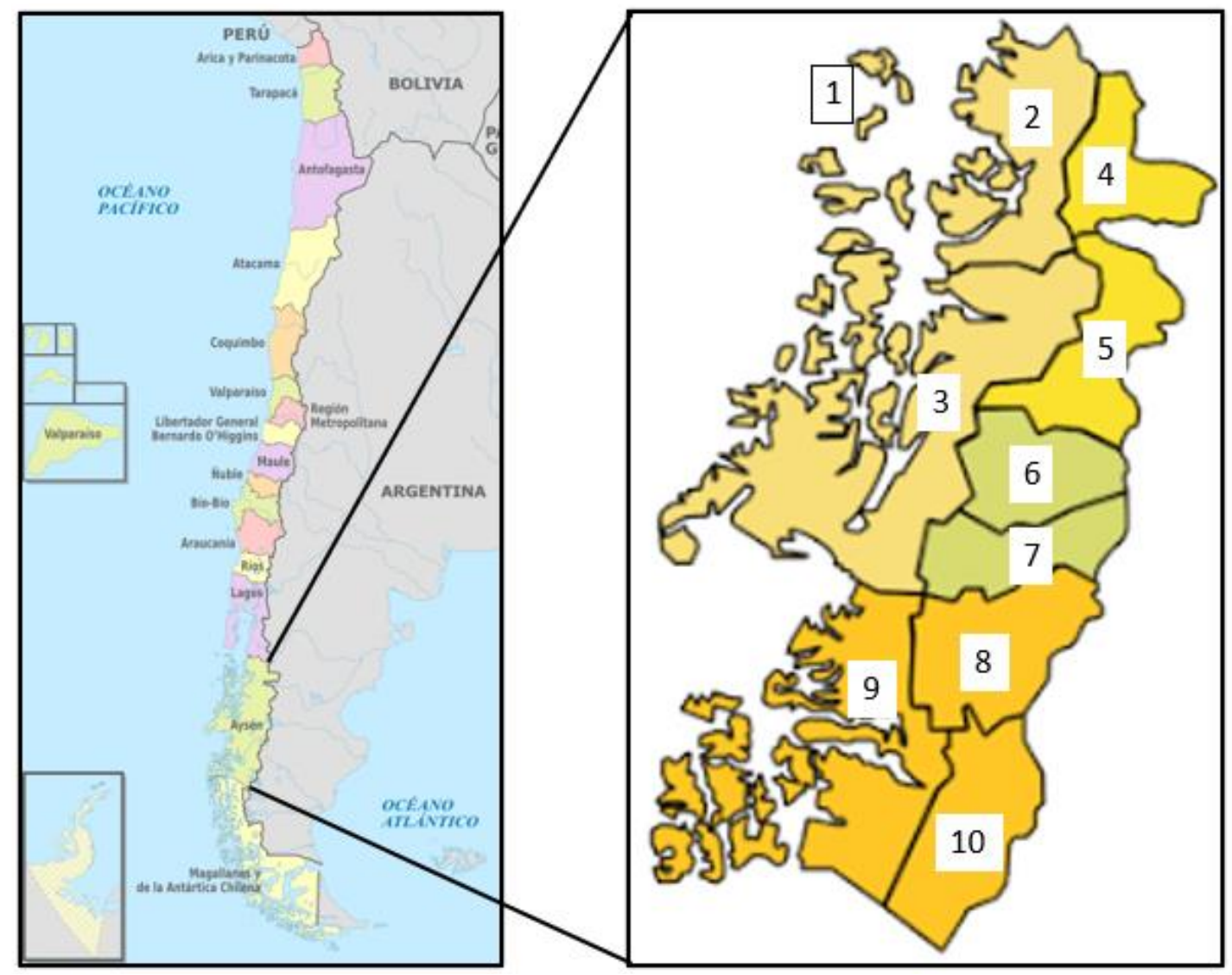

Figura. 1. Ubicación de la Región de Aysén (izquierda) en Chile. Región de Aysén (derecha) Provincia de Aisén: Comunas de Guaitecas (1), Cisnes (2) y Aisén (3); Provincia de Coyhaique: Comunas de (Lago Verde (4) y Coyhaique (5); Provincia de General Carrera: Comunas de Río Ibáñez (6) y Chile Chico (7); Provincia de Capitán Prat (Lugar de trabajo): Comunas de Cochrane (8), Tortel (9) y O’Higgins (10). La localización de los censos de vegetación levantados en el lugar de trabajo se presenta en Anexo. Figuras modificadas de colored_2018.svg (izquierda) y de Guía Turística de la Región de Aisén, Servicio Nacional de Turismo 1991 (derecha).

Location of Aysén Region (left) in Chile. Region of Aysén (right) Province of Aisén: Communes of Guaitecas (1), Cisnes (2) and Aisén (3); Province of Coyhaique: Communes of (Lago Verde (4) and Coyhaique (5); Province of General Carrera: Communes of Río Ibañez (6) and Chile Chico (7); Province of Capitán Prat (work place): Communes of Cochrane (8), Tortel (9) and O'Higgins (10) The geographical location of the vegetation samples carried out in the workplace are presented in the Annex. Figures modified from colored_2018.svg (left) and from the Aisén Region Tourist Guide, National Tourism Service (SERNATUR) 1991 (right).

entre las comunidades utilizando los índices de Jaccard y de Ellenberg; el primero sólo considera el número de especies y el otro pondera el promedio de cobertura, es decir, la abundancia. Las diferencias de similitud encontradas fueron representadas en una constelación de comunidades.

Estadística multivariada. Para obtener mayor cantidad de información ecológica de la tabla fitosociológica inicial se aplicaron métodos estadísticos multivariados de clasificación y ordenación. Como ha sido mencionado con frecuencia (Clarke 1993, San Martín et al. 1999, Sandoval et al. 2016) los análisis estadísticos multivariados de ordenación, tanto de censos como de especies, permiten inferir requerimientos de sitio utilizando las especies de los extremos del arreglo, que por ser definidas en sus preferencias y reconocidas empíricamente en sus requerimientos de sitio, permiten definir a que factor ecológico corresponderían los primeros ejes del ordenamiento, lo que facilita inferir los requerimientos de las especies desconocidas en relación con las especies conocidas. En vegetación de tipo zonal los primeros ejes corresponden principalmente a requerimientos hídricos, térmicos y lumínicos y luego vendrían los requerimientos edáficos, que serían importantes solo en vegetación de tipo azonal (San Martín et al. 2014a).

El análisis de conglomeración o clasificación se llevó a cabo mediante el uso del método jerárquico flexible beta con la distancia de Bray-Curtis y un parámetro $\beta=-0,25$ (McCune y Grace 2002) previa transformación de los valores de abundancia a logaritmo log (abundancia +1$)$.

La ordenación de censos y especies se realizó con un Análisis Multidimensional No Métrico o NMMS (Non Metric Muldimensional Scaling) para obtener una confi- 
guración gráfica de la ubicación de las especies vegetales y de las unidades de muestreo en los dos primeros ejes extraídos, visualizando la relación entre los sitios en función de la composición florística.

\section{RESULTADOS}

Flora. La flora de todas las comunidades turbosas estudiadas está formada por 106 especies, de ellas 102 son vasculares y cuatro no vasculares, Sphagnum magellanicum, S. fimbriatum, Racomitrium lanuginosum (musgos) y Cladonia rangiferina (líquen); además se agregó una denominación colectiva para el resto de los musgos. De las plantas vasculares solo tres son especies alóctonas introducidas (Festuca rubra, Juncus planifolius y Poa bulbosa); en el resto, 99 especies corresponden a plantas nativas, de las cuales, solo una es endémica de Chile (Lepidothamnus fonkii). Entre las restantes, 90 se comparten con Argentina, dos (Austrolycopodium magellanicum y Blechnum pennamarina) con Argentina y Bolivia y cuatro (Desfontainia fulgens, Gunnera magellanica, Myrteola nummularia y Oreobolus obtusangulus) con Argentina, Bolivia y Perú.

Determinación de comunidades vegetales. La tabla inicial formada por 106 especies (filas) y 204 censos (columnas) fue ordenada mediante la búsqueda de especies diferenciales, obteniéndose cuatro grupos (comunidades o asociaciones vegetales), el primero con 49 censos, el segundo con 77 , el tercero con 40 y el último con 38 censos (cuadro 1). El primer grupo fue diferenciado por la presencia de Sphagnum magellanicum y Empetrum rubrum, el segundo por Pilgerodendron uviferum y Lepidothamnus fonkii, el tercero por Schoenus andinus y Cortaderia egmontiana y el último por Donatia fascicularis y Astelia pumila. Ninguna de estas especies diferenciales se comportó como exclusiva, por el contrario, todas ellas están presentes en los otros grupos incluso, Lepidothamnus fonkii apareció con alta frecuencia, presentándose en 32 de 38 censos con una cobertura total de $1.207 \%$ en el último grupo de censos. Lo mismo sucedió con Sphagnum magellanicum que se pre- sentó en 10 de 77 censos con una cobertura total de $748 \%$ ben el segundo grupo.

Descripción de las comunidades encontradas. Los grupos encontrados corresponden a comunidades o asociaciones vegetales poco conocidas, por lo cual se entrega una breve descripción de cada una, de acuerdo a los resultados, a lo actualmente conocido y encontrado en la literatura pertinente, que se cita y a observaciones en terreno.

- Gaultherio-Sphagnetum magellanici Oberdorfer 1960, (Turbera esfagnosa de Sphagnum magellanicum, comunidad de Sphagnum magellanicum, Mallín colorado, Turbera de pon-pon, Sphagnum bog).

Las turberas de Sphagnum magellanicum son pantanos fríos de lugares planos en bajas altitudes, generalmente junto a cursos de agua, sin drenaje y con minerotrofía aportada por agua edáfica. Presentan condiciones extremas de anegamiento y de anaerobiosis (San Martín et al. 2018). El sustrato de turba presenta alta acidez. El sustrato suele superar el metro de espesor y está formado por restos orgánicos en lenta descomposición de las especies de la cubierta vegetal, por la anoxia que entrega la saturación del sustrato sin que haya circulación de agua (Frahm 2001). En la Región de Aisén estas turberas son abundantes en tierras bajas.

En los 49 censos levantados se encontraron 64 especies, con un promedio de 10,9 especies por censo. Las más importantes son Sphagnum magellanicum, Empetrum rubrum y Marsippospermum grandiflorum, la primera está presente en todos los censos con un promedio de $79 \%$ de cobertura, la segunda solo faltó en dos censos y presenta una cobertura promedio de $20 \%$ y la tercera, aparece en 24 censos con un promedio de $9 \%$ de cobertura por censo. La última especie aumenta en años secos, desplazando a Sphagnum magellanicum. También son importantes, aunque con menores valores de frecuencia y cobertura, $\mathrm{Te}$ troncium magellanicum, Pilgerodendron uviferum, Carex magellanica y Myrteola nummularia.

Cuadro 1. Riqueza florística de las distintas comunidades turbosas trabajadas.

Floristic richness of the different worked peat bog communities.

\begin{tabular}{|c|c|c|c|}
\hline Comunidad & Censos & Especies & Promedio especies / censos \\
\hline Sphagnum magellanicum & 49 & 64 & 10,9 \\
\hline Lepidothamnus fonkii & 77 & 79 & 13,2 \\
\hline Cortaderia-Schoenus & 40 & 71 & 14,1 \\
\hline Donatia fascicularis & 38 & 55 & 12,1 \\
\hline Total & 204 & $*$ & 12,58 \\
\hline
\end{tabular}

*varias especies se repiten. 
Estas turberas son de color rojizo en verano por la presencia de esfagnorrubina en Sphagnum y presentan una superficie ondulada, a veces con grandes oquedades de agua libre, en cuyas orillas encuentra lugar de vida Tetroncium magellanicum, actuando como una especie acuática. Por sobre la superficie se levantan subarbustos de Empetrum rubrum. Es importante la presencia de árboles que alcanzan tamaños reducidos por las condiciones extremas del hábitat, por ejemplo, Pilgerodendron uviferum, Nothofagus antarctica y $N$. betuloides. También destacan grandes poblaciones del helecho Blechnum penna-marina que en primavera y verano suelen presentarse quemadas, posiblemente por exceso de radiación solar (Ramírez et al. 2018)

- Schoeno andinus-Lepidothamnetum fonkii Ramírez et al. 2014b (Turbera esfagnosa de Lepidothamnus fonkii, comunidad de ciprés enano, Mallín negro).

La turbera de ciprés enano es una comunidad con requerimientos de sitio parecida a los turbales esfagnosos, pero por observaciones en terreno, coloniza terrenos planos, anegados en altitudes intermedias y mayores dificultades en el drenaje lo que permite el desarrollo importante del ciprés de las Guaitecas, que suele formar bosquetes que con menor nivel freático se transforman en comunidades boscosas de ciprés de Las Guaitecas (Pilgerodendronetum uviferi Oberdorfer 1960), disminuyendo su condición turbosa.

En los 77 censos levantados se encontraron 79 especies con un promedio de 13,2 por censo. La más frecuente es Lepidothamnus fonkii, presente en todos los censos con cobertura promedio de $64 \%$, seguida por Sphagnum magellanicum, presente en 34 de 77 censos con un promedio de $22 \%$ de cobertura y Pilgerodendron uviferum presente en 59 de 77 censos pero con menor cobertura promedio $(8 \%)$. Esta última especie aparece formando pequeñas islas dentro de la turbera. Con frecuencias superiores a la mitad de los censos, pero con menor cobertura promedio siguen Schoenus andinus, Donatia fascicularis, Astelia pumila y Nothofagus betuloides.

Las turberas de ciprés enano son ricas en especies y tienen un estrato muscinal formado por Sphagnum magellanicum sobre el cual se levantan arbustos como Pilgerodendron uviferum, Gaultheria pumila, G. mucronata, Empetrum rubrum y la hierba alta Marsippospermum grandiflorum, que suele otorgarle un aspecto estepario, desmentido por el nivel de saturación siempre presente. Presentan un color pardo muy oscuro, por lo que son fácilmente diferenciables de aquellas de pon-pon que son rojizas.

- Cortaderio egmontianae-Schoenetum andinus asoc. nova. (Turbera graminosa de Cortaderia egmontiana y Schoenus andinus, comunidad de Cortaderia y Schoenus, Turbal graminoso de Quilmén, Mallines amarillos).
Las turberas graminosas de Schoenus andinus tienen un color amarillento y parecen un coironal estepario, pero se trata de una comunidad de turbera que crece en la cima y laderas de cerros más bajos que forman la cordillera, en altitud media. Ellas están rodeadas de una matriz de bosque magallánico perennifolio (Nothofagetum betuloidis Oberdorfer 1960) que se extiende por las quebradas más húmedas y abrigadas. En terreno se aprecia que la capa de turba es delgada, hasta $50 \mathrm{~cm}$ de espesor como máximo y generalmente colonizada por pequeños árboles y arbustos.

En los 40 censos levantados se encontró un total de 71 especies con un promedio de 14,1 especies por censo. La especie más importante (V.I. (Valor de Importancia) $=52$ ) resultó ser Schoenus andinus presente en todos los censos con una cobertura total de $2.095 \%$. Esta planta pertenece a la familia ciperáceas, presenta unos $40 \mathrm{~cm}$ de altura con tallos amarillentos y una espiga terminal de color negro. Le siguen Cortaderia egmontiana "coirón" presente en 37 censos con una cobertura total menor $(865 \%)$ y Marsippospermum grandiflorum juncácea con aspecto de Junquillo grueso, de aproximadamente $40 \mathrm{~cm}$ de altura que aparece en 17 censos con una frecuencia de $43 \%$ y una cobertura total que alcanza solo a $229 \%$, la primera tiene un valor de importancia de 25,16 y la segunda de 7,94. Lepidothamnus fonkii presenta aquí baja frecuencia (20) y cobertura total (110\%) (cuadro 2). Entre los árboles presentes con individuos de poca altura, se encuentran Nothofagus betuloides, $N$. antarctica, Embothrium coccineum y Pilgerodendron uviferum. En el mismo cuadro se muestra el censo número 23 de la tabla parcial de esta comunidad, que se propone como censo tipo para esta nueva asociación vegetal.

Estas turberas graminosas presentan tres estratos, uno con hierbas camefíticas, otro de hierbas altas (ciperáceas, juncáceas y poáceas) y, por último, un estrato arbustivo/ arbóreo con arbolitos de hasta $4 \mathrm{~m}$, entre los que destaca Embothrium coccineum. Un bajo porcentaje de turba, que aparece desnuda o cubierta por musgos, le da un color pardo oscuro y los pastos, ciperáceas y juncáceas, un color amarillento. Esta turbera es ombrogénica y normalmente no presenta anegamiento superficial.

- Drosero uniflorae-Donatietum fascicularis Ramírez 1968 (Turbera pulvinada de Drosera uniflora y Donatia fascicularis, comunidad de Donatia fascicularis, Turbera de Donatia, Pampas verdes, Donatia bog).

Ocupa los lugares de mayor altitud, secos e inclinados en comparación con las tres comunidades anteriormente descritas, se presenta con una cubierta continua de hierbas pulviniformes (en cojín) sobre la que crecen escasos arbustos enanos. Los cojines, aunque blandos son secos y se puede caminar fácilmente y con seguridad sobre ellos. Entre su verdor, destaca el tono rojizo de la pequeña insectívora Drosera uniflora (San Martín et al. 2004) 
Cuadro 2. Resumen de la tabla parcial con 40 censos y 70 especies de la nueva asociación vegetal turbosa Cortaderio egmontianaeSchoenetum andinus propuesta en este trabajo. El censo 23 (segunda columna) de la tabla parcial de la comunidad fue designado como censo tipo. Abreviaturas: Frec. $=$ frecuencia absoluta, Frec.Rel. $=$ Frecuencia relativa, Cob. Total $=$ Cobertura total, Cob. Rel. $=$ Cobertura relativa, V.I. $=$ Valor de importancia.

Summary of the partial table with 40 vegetation samples and 70 plant species of the new bog plant association Cortadero egmontianaeSchoenetum andinus proposed in this work. The vegetation sample number 23 (second column) of the partial table was considered as type for the plant association. Abbreviations: Frec. $=$ Absolute frequency, Frec.Rel $=$ Relative frequency, Cob. Total $=$ Total coverage, Cob.Rel. $=$ Relative coverage. V.I. Importance value.

\begin{tabular}{|c|c|c|c|c|c|c|}
\hline \multirow{2}{*}{ Especies } & \multirow{2}{*}{$\begin{array}{c}\text { Censo tipo } \\
23\end{array}$} & \multicolumn{5}{|c|}{ Cálculo del valor de valor de importancia } \\
\hline & & Frec. & Frec.Rel. & Cob.Total & Cob.Rel. & V.I. \\
\hline Schoenus andinus & 50 & 40 & 7,10 & 2.095 & 45,02 & 52,12 \\
\hline Cortaderia egmontiana & 40 & 37 & 6,57 & 865 & 18,59 & 25,16 \\
\hline Musgos & 10 & 25 & 4,44 & 193 & 4,15 & 8,59 \\
\hline Marsippospermum grandiflorum & & 17 & 3,02 & 229 & 4,92 & 7,94 \\
\hline Astelia pumila & & 18 & 3,20 & 159 & 3,42 & 6,61 \\
\hline Lepidothamnus fonkii & & 20 & 3,55 & 110 & 2,36 & 5.92 \\
\hline Myrteola nummularia & 1 & 20 & 3,55 & 85 & 1,83 & 5,38 \\
\hline Chilliotrichum diffusum & 1 & 21 & 3,73 & 65 & 1,40 & 5,13 \\
\hline Donatia fascicularis & & 20 & 3,55 & 54 & 1,16 & 4,71 \\
\hline Hypochaeris palustris & 1 & 20 & 3,55 & 33 & 0,71 & 4,26 \\
\hline Philesia magellanica & & 16 & 2,84 & 43 & 0,92 & 3,77 \\
\hline Nothofagus betuloides & & 15 & 2,66 & 33 & 0,71 & 3,37 \\
\hline Blechnum penna-marina & & 14 & 2,49 & 41 & 0,88 & 3,37 \\
\hline Nothofagus antarctica & 1 & 14 & 2,49 & 31 & 0,67 & 3,15 \\
\hline Drosera uniflora & & 15 & 2,66 & 21 & 0,45 & 3,12 \\
\hline Oreobolus obtusangulus & & 15 & 2.66 & 15 & 0,32 & 2,99 \\
\hline Poa vulcanica & & 9 & 1,60 & 56 & 1,20 & 2,80 \\
\hline Tetroncium magellanicum & 1 & 10 & 1,78 & 42 & 0,90 & 2,68 \\
\hline Baccharis patagonica & 1 & 10 & 1,78 & 41 & 0,88 & 2,66 \\
\hline Acaena pumila & 1 & 10 & 1,78 & 38 & 0,82 & 2,59 \\
\hline Sphagnum magellanicum & 1 & 7 & 1,24 & 53 & 1,14 & 2.38 \\
\hline Embothrium coccineum & 1 & 11 & 1,95 & 15 & 0,32 & 2,28 \\
\hline Gaultheria mucronata & & 10 & 1,78 & 21 & 0,45 & 2,23 \\
\hline Gaultheria antarctica & 1 & 11 & 1,95 & 11 & 0,24 & 2,19 \\
\hline Gaultheria pumila & & 9 & 1,60 & 27 & 0,58 & 2,18 \\
\hline Blechnum magellanicum & & 10 & 1,78 & 10 & 0,21 & 1,99 \\
\hline Berberis microphylla & & 9 & 1,60 & 9 & 0,19 & 1,79 \\
\hline Caltha appendiculata & & 9 & 1,60 & 9 & 0,19 & 1,99 \\
\hline Cladonia rangiferina & & 9 & 1,60 & 9 & 0,19 & 1,79 \\
\hline Pilgerodendron uviferum & & 5 & 0,89 & 37 & 0,80 & 1,68 \\
\hline Gunnera magellanica & & 7 & 1,24 & 11 & 0,24 & 1,48 \\
\hline Symphyotrichum vahlii & 1 & 7 & 1,24 & 8 & 0,17 & 1,42 \\
\hline Berberis ilicifolia & & 7 & 1,24 & 7 & 0,15 & 1,39 \\
\hline
\end{tabular}


Cuadro 2. Continuación

\begin{tabular}{|c|c|c|c|c|c|c|}
\hline Austrolycopodium confertum & & 5 & 0,89 & 19 & 0,41 & 1,30 \\
\hline Luzuriaga marginata & & 6 & 1,07 & 6 & 0,13 & 1,19 \\
\hline Senecio triodon & & 5 & 0,89 & 8 & 0,17 & 1,06 \\
\hline Austrolycopodium magellanicum & & 4 & 0,71 & 12 & 0,26 & 0,97 \\
\hline Acaena magellanica & & 4 & 0,71 & 8 & 0,17 & 0,88 \\
\hline Caltha dioneifolia & 1 & 4 & 0,71 & 8 & 0,17 & 0.88 \\
\hline Macrachaenium gracile & & 4 & 0,71 & 8 & 0,17 & 0,88 \\
\hline Carex magellanica & & 2 & 0,36 & 21 & 0,45 & 0,81 \\
\hline Sticherus quadripartitus & & 3 & 0,53 & 7 & 0,15 & 0,68 \\
\hline Azorella trifurcata & & 1 & 0,18 & 20 & 0,43 & 0,61 \\
\hline Gunnera lobata & & 3 & 0,53 & 3 & 0,06 & 0,60 \\
\hline Lagenophora hariotii & & 3 & 0,53 & 3 & 0,06 & 0,60 \\
\hline Perezia magellanica & & 3 & 0,53 & 3 & 0,06 & 0,60 \\
\hline Pinguicula antarctica & & 3 & 0,53 & 3 & 0,06 & 0,60 \\
\hline Senecio acanthifolius & & 3 & 0,53 & 3 & 0,06 & 0,60 \\
\hline Tapeinia magellanica & & 3 & 0,53 & 3 & 0,06 & 0,60 \\
\hline Hypochaeris tenuifolia & & 2 & 0,36 & 6 & 0,13 & 0,48 \\
\hline Drimys winteri & & 2 & 0,36 & 2 & 0,04 & 0,40 \\
\hline Empetrum rubrum & & 2 & 0,36 & 2 & 0,04 & 0,40 \\
\hline Festuca rubra & & 2 & 0,36 & 2 & 0,04 & 0,40 \\
\hline Nanodea muscosa & & 2 & 0,36 & 2 & 0,04 & 0,40 \\
\hline Perezia pilifera & & 2 & 0,36 & 2 & 0,04 & 0,40 \\
\hline Phyllachne uliginosa & & 2 & 0,36 & 2 & 0,04 & 0,40 \\
\hline Valeriana lapathifolia & & 2 & 0,36 & 2 & 0,04 & 0,40 \\
\hline Festuca purpurascens & & 1 & 0,18 & 10 & 0,21 & 0,39 \\
\hline Acaena antarctica & & 1 & 0,18 & 1 & 0,02 & 0,20 \\
\hline Baccharis magellanica & & 1 & 0,18 & 1 & 0,02 & 0,20 \\
\hline Escallonia alpina & & 1 & 0,18 & 1 & 0,02 & 0,20 \\
\hline Escallonia virgata & & 1 & 0,18 & 1 & 0,02 & 0,20 \\
\hline Griselinia ruscifolia & & 1 & 0,18 & 1 & 0,02 & 0,20 \\
\hline Myoschilos oblonga & & 1 & 0,18 & 1 & 0,02 & 0,20 \\
\hline Nothofagus pumilio & & 1 & 0,18 & 1 & 0,02 & 0,20 \\
\hline Ortachne rariflora & & 1 & 0,18 & 1 & 0,02 & 0,20 \\
\hline Perezia lactucoides & & 1 & 0,18 & 1 & 0,02 & 0,20 \\
\hline Poa resinulosa & & 1 & 0,18 & 1 & 0,02 & 0,20 \\
\hline Ranunculus spoegazzinii & & 1 & 0,18 & 1 & 0,02 & 0,20 \\
\hline Selenomelus segethii & & 1 & 0,18 & 1 & 0,02 & 0,20 \\
\hline Tribeles australis & & 1 & 0,18 & 1 & 0,02 & 0,20 \\
\hline 70 Especies en total / Totales: & 15 & 563 & 100 & 4.654 & 100 & 200 \\
\hline
\end{tabular}


De esta comunidad se levantaron 38 censos que reunieron un total de 55 especies vegetales con un promedio de 12,1 especies por censo. La especie con mayor valor de importancia $(36,03)$ fue Donatia fascularis y aunque faltó en tres censos, presentó la más alta cobertura total $1.380 \%$. Le siguen Lepidothamnus fonkii con una frecuencia de 32 ( $84 \%$ ) y una cobertura total de $1.207 \%$ y en tercer lugar se presenta Astelia pumila, una pequeña hierba acojinada de cortas hojas rígidas y punzantes con la más alta frecuencia (38 censos y $100 \%$ de frecuencia), siendo una buena especie diferencial de esta comunidad. La primera especie tiene un valor de importancia de 31,82 y la segunda de 28,38. La insectívora Drosera uniflora está presente en 30 de 38 censos y tiene una cobertura total de $147 \%$.

Esta turbera presenta escasas oquedades con agua libre, tiene una capa de turba que puede alcanzar hasta 1 $\mathrm{m}$ de espesor y es típicamente ombrotrófica, dependiendo solamente del agua de lluvia. Su superficie es ondulada y continua, ubicándose generalmente en pendientes suaves (San Martín et al. 2004).

Comparación de las turberas diferenciadas. El número de especies de las turberas fue diferente, aunque se presentó correlación positiva $\left(\mathrm{r}=0,78 ; \mathrm{R}^{2}=0,609\right)$ entre el número de censos levantados en cada una y el número de ellas (cuadro 1). En todo caso el promedio de especies por censo fue mayor en la turbera graminosa de Cortaderia y Schoenus $(14,1)$ y el menor en la esfagnosa de Sphagnum magellanicum $(10,9)$ que correspondería a la comunidad con hábitat más extremo. Las otras dos comunidades tienen valores intermedios. Estas dos comunidades (de Cortaderia schoenus y de Sphagnum magellanicum) junto con la turbera de Donatia tienen histogramas de frecuencia propios de una asociación vegetal bien homogénea, definida y limitada. No sucede lo mismo con la
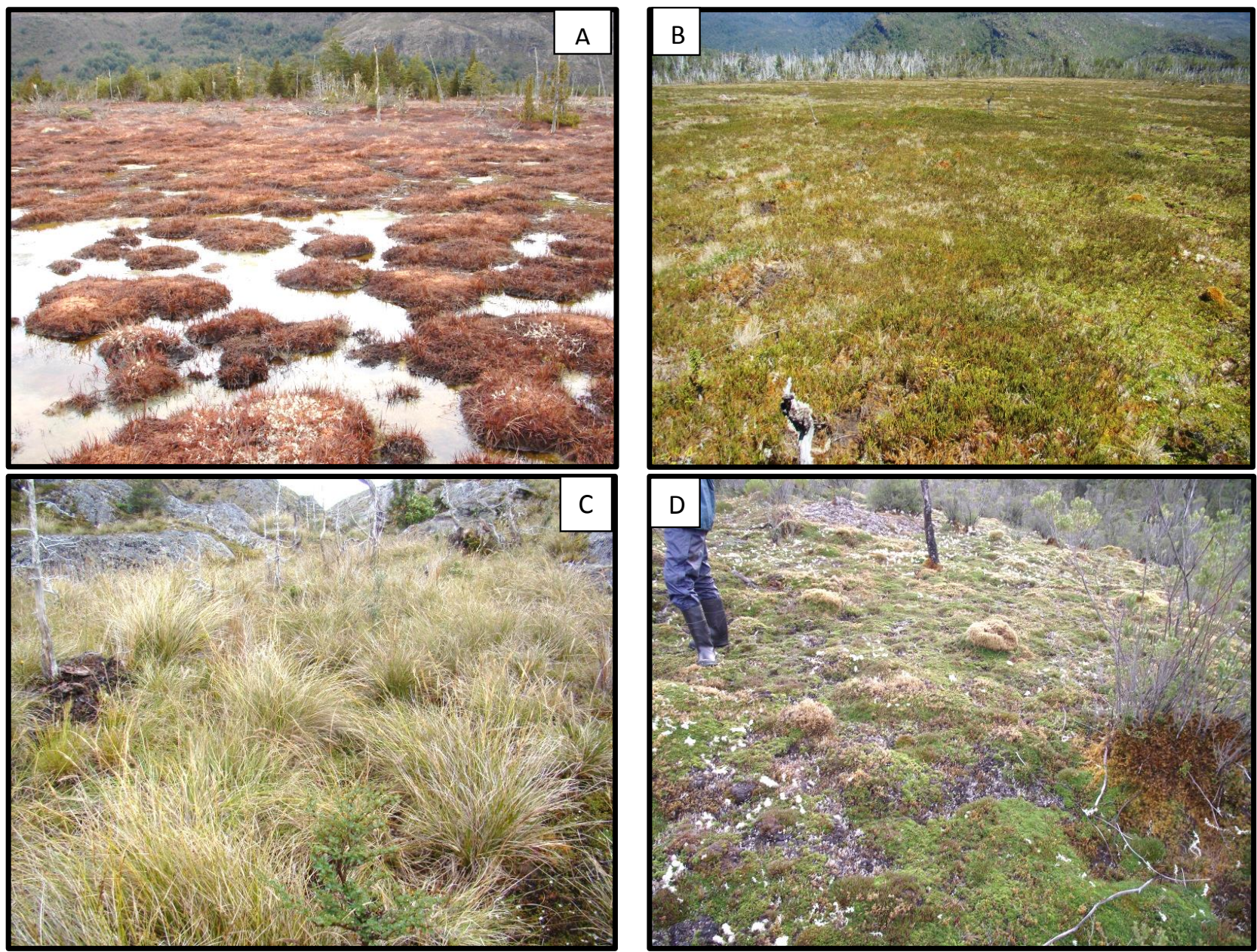

Figura 2. $\mathrm{A}=$ Comunidad turbosa esfagnosa de Sphagnum magellanicum, $\mathrm{B}=$ Comunidad turbosa esfagnosa de Lepidothamnus fonkii, $\mathrm{C}$ $=$ Comunidad turbosa graminosa de Cortaderia - Schoenus andinus, $\mathrm{D}=$ Comunidad turbosa pulvinada de Drosera-Donatia fascicularis .

$\mathrm{A}=$ Peat bog community of Sphagnum magellanicum, B = Peat bog community of Lepidothmanus fonkii, $\mathrm{C}=$ Peat bog community of Cortaderia -Schoenus andinus, D = Peat bog community of Drosera - Donatia fascicularis. 
Cuadro 3. Coberturas promedio de las especies presentes en las distintas comunidades vegetales turbosas descritas en la Región de Aisén (Chile) ordenadas por frecuencia (Fr.) y cobertura total (Cob.Total). Abreviaturas de las comunidades: Sm $=$ de Sphagnum magellanicum, $\mathrm{Lf}=$ de Lepidothamnus fonkii, C-Sa = Comunidad de Cortaderia - Schoenus andinus, D-Df = de Drosera - Donatia fascicularis.

Cover average of the species in the different peat bog plant communities described in Aisén Region (Chile) sorted by frequency (Fr.) and total coverage (Cob.Total). Abreviations of communities: $\mathrm{Sm}=$ community of Sphagnum magellanicum, $\mathrm{Lf}=$ community of Lepidothamnus fonkii, $\mathrm{C}-\mathrm{Sa}=$ community of Cortaderia - Schoenus andinus, D-Df = community of Drosera - Donatia fascicularis.

\begin{tabular}{|c|c|c|c|c|c|}
\hline \multirow{2}{*}{ Especies Autor } & \multicolumn{4}{|c|}{ Comunidad } & \multirow[b]{2}{*}{ Fr. } \\
\hline & $\mathrm{Sm}$ & Lf & $\mathrm{C}-\mathrm{Sa}$ & D-Df & \\
\hline Lepidothamnus fonkii Phil. & 1 & 64 & 3 & 32 & 4 \\
\hline Sphagnum magellanicum Brid. & 79 & 10 & 1 & 1 & 4 \\
\hline Schoenus andinus (Phil.) H. Pfeiff. & 1 & 4 & 52 & 1 & 4 \\
\hline Donatia fascicularis J.R. Forst. et G. Forst. & 1 & 4 & 1 & 36 & 4 \\
\hline Astelia pumila (G. Forst.) Gaudich. & 1 & 4 & 4 & 26 & 4 \\
\hline Musci & 2 & 10 & 5 & 13 & 4 \\
\hline Cortaderia egmontiana (Roem. et Schult.) M. Lyle ex Connor & 1 & 1 & 22 & 1 & 4 \\
\hline Empetrum rubrum Vahl ex Willd. & 20 & 1 & 1 & 1 & 4 \\
\hline Marsippospermum grandiflorum (L. f) Hook. f. & 9 & 1 & 6 & 1 & 4 \\
\hline Pilgerodendron uviferum (D. Don) Florin & 4 & 6 & 1 & 1 & 4 \\
\hline Tetroncium magellanicum Willd. & 4 & 2 & 1 & 2 & 4 \\
\hline Nothofagus betuloides (Mirb.) Oerst. & 3 & 3 & 1 & 1 & 4 \\
\hline Drosera uniflora Willd. & 1 & 1 & 1 & 4 & 4 \\
\hline Oreobolus obtusangulus Gaudich. & 1 & 4 & 1 & 1 & 4 \\
\hline Carex magellanica Lam. & 2 & 1 & 1 & 2 & 4 \\
\hline Myrteola nummularia (Poir.) O. Berg. & 2 & 1 & 2 & 1 & 4 \\
\hline Nothofagus antarctica (G. Forst.) Oerst. & 3 & 1 & 1 & 1 & 4 \\
\hline Chilliotrichum diffusum (G. Forst.) Kuntze & 1 & 1 & 2 & 1 & 4 \\
\hline Gaultheria mucronata (L. f.) Hook et Arn. & 2 & 1 & 1 & 1 & 4 \\
\hline Berberis ilicifolia L. f. & 1 & 1 & 1 & 1 & 4 \\
\hline Berberis microphylla G. Forst. & 1 & 1 & 1 & 1 & 4 \\
\hline Caltha appendiculata Pers. & 1 & 1 & 1 & 1 & 4 \\
\hline Caltha dioneifolia Hook. f. & 1 & 1 & 1 & 1 & 4 \\
\hline Cladonia rangiferina (L.) Weber ex F. H. Wigg. & 1 & 1 & 1 & 1 & 4 \\
\hline Embothrium coccineum J. R. Forst. et G. Forst. & 1 & 1 & 1 & 1 & 4 \\
\hline Festuca purpurascens Bank. et Sol. ex Hook. f. & 1 & 1 & 1 & 1 & 4 \\
\hline Festuca rubra L. & 1 & 1 & 1 & 1 & 4 \\
\hline Gaultheria antarctica Hook. f. & 1 & 1 & 1 & 1 & 4 \\
\hline Gaultheria pumila (L. f.) D.J. Middleton & 1 & 1 & 1 & 1 & 4 \\
\hline Gunnera magellanica Hook. f. & 1 & 1 & 1 & 1 & 4 \\
\hline Hypochaeris palustris (Phil.) Reiche & 1 & 1 & 1 & 1 & 4 \\
\hline Luzuriaga marginata (Gaernt.) Benth. et Hook. f. & 1 & 1 & 1 & 1 & 4 \\
\hline Austrolycopodium confertum Willd. & 1 & 1 & 1 & 1 & 4 \\
\hline Austrolycopodium magellanicum (P. Beauv.) Sw. & 1 & 1 & 1 & 1 & 4 \\
\hline
\end{tabular}


Myoschilos oblonga Ruiz et Pav.

Nanodea muscosa Banks. et C.F. Gaertn.

Philesia magellanica G. F. Gmel.

Tapeinia pumila (G. Forst.) Baill.

Tribeles australis Phil.

Baccharis patagónica Hook. et Arn.

Blechnum penna-marina (Poir.) Kuhn

Drimys winteri J.R. Forst. et G. Forst.

Gaimardia australis Gaudich.

Racomitrium lanuginosum (Hedw.) Brid.

Sphagnum fimbriatum Wilson

Acaena pumila Vahl.

Gunnera lobata Hook. f.

Lagenophora hariotii Franchet

Ortachne rariflora (Hook. f.) Hughes

Perezia magellanica (L. f.) Less.

Phyllachne uliginosa J. R. Forst. et G. Forst,

Poa resinulosa Nees ex Steud.

Senecio acanthifolius Hombr. et Jacquinot

Hymenophyllum dentatum Cav.

Hymenophyllum secundum Hook. et Grev.

Escallonia virgata (Ruiz et Pav.) Pers.

Symphyotrichum vahlii (Gaudich.) G.L. Nesom

Blechnum magellanicum (Dresv.) Mett.

Azorella trifurcata (Gaertn.) Pers.

Pinguicula antarctica Vahl.

Poa bulbosa L.

Senecio triodon Phil.

Acaena antarctica Hook. f.

Sticherus quadripartita (Poir.) Ching.

Hypochaeris tenuifolia (Hook. et Arn.) Crev. ex Griseb.

Macrachaenium gracile Hook. f.

Perezia lactucoides (Vahl.) Less.

Bolax gummifera (Lam.) Spreng.

Rostkovia magellanica (Lam.) Hook. f.

Azorella ranunculus d'Urv.

Perezia pilifera (D. Don) Hook. et Arn.

Raukaua laetevirens (Gay) Frodin

Ribes magellanicum Poir.

Rubus geoides $\mathrm{Sm}$.
Cuadro 3. Continuación

\begin{tabular}{|c|c|c|c|c|}
\hline 1 & 1 & 1 & 1 & 4 \\
\hline 1 & 1 & 1 & 1 & 4 \\
\hline 1 & 1 & 1 & 1 & 4 \\
\hline 1 & 1 & 1 & 1 & 4 \\
\hline 1 & 1 & 1 & 1 & 4 \\
\hline 1 & 1 & 1 & & 3 \\
\hline 1 & 1 & 1 & & 3 \\
\hline 1 & 1 & 1 & & 3 \\
\hline 1 & 1 & & 1 & 3 \\
\hline 1 & 1 & & 1 & 3 \\
\hline \multirow[t]{9}{*}{1} & 1 & & 1 & 3 \\
\hline & 1 & 1 & 1 & 3 \\
\hline & 1 & 1 & 1 & 3 \\
\hline & 1 & 1 & 1 & 3 \\
\hline & 1 & 1 & 1 & 3 \\
\hline & 1 & 1 & 1 & 3 \\
\hline & 1 & 1 & 1 & 3 \\
\hline & 1 & 1 & 1 & 3 \\
\hline & 1 & 1 & 1 & 3 \\
\hline 1 & 1 & & & 2 \\
\hline 1 & 1 & & & 2 \\
\hline 1 & & 1 & & 2 \\
\hline 1 & & 1 & & 2 \\
\hline 1 & & 1 & & 2 \\
\hline \multirow[t]{13}{*}{1} & & & 1 & 2 \\
\hline & 1 & 1 & & 2 \\
\hline & 1 & 1 & & 2 \\
\hline & 1 & 1 & & 2 \\
\hline & 1 & 1 & & 2 \\
\hline & 1 & 1 & & 2 \\
\hline & 1 & 1 & & 2 \\
\hline & 1 & 1 & & 2 \\
\hline & 1 & 1 & & 2 \\
\hline & 1 & & 1 & 2 \\
\hline & 1 & & 1 & 2 \\
\hline & & 1 & 1 & 2 \\
\hline & & 1 & 1 & 2 \\
\hline 1 & & & & 1 \\
\hline 1 & & & & 1 \\
\hline 1 & & & & 1 \\
\hline
\end{tabular}

Contunúa 
Cuadro 3. Continuación

\begin{tabular}{|c|c|c|c|c|c|}
\hline Isolepis inundata $\mathrm{R} . \mathrm{Br}$. & 1 & & & & 1 \\
\hline Hymenophyllum pectinatum Cav. & 1 & & & & 1 \\
\hline Juncus planifolius $\mathrm{R}$. Br. & 1 & & & & 1 \\
\hline Juncus procerus E. Mey. & 1 & & & & 1 \\
\hline Bolax caespitosa Hombr. et Jacquinot & 1 & & & & 1 \\
\hline Carex brongniartii Kunth & 1 & & & & 1 \\
\hline Amomyrtus luma (Mol.) D. Legrand et Kausel & 1 & & & & 1 \\
\hline Eleocharis pachycarpa E. Desv. & 1 & & & & 1 \\
\hline Nertera granadensis (Mutis ex L. f.) Druce & 1 & & & & 1 \\
\hline Poa obvallata Steud. & 1 & & & & 1 \\
\hline Escallonia rosea Griseb. & & & 1 & & 1 \\
\hline Gaultheria caespitosa Poepp. et Endl. & & & 1 & & 1 \\
\hline Chloraea magellanica Hook. f. & & & 1 & & 1 \\
\hline Lebethantus myrsinites (Lam.) Dusén & & & 1 & & 1 \\
\hline Lomatia ferruginea (Cav.) R. Br. & & & 1 & & 1 \\
\hline Austrolycopodium albolffi Rolleri & & & 1 & & 1 \\
\hline Misodendron punctulatum Banks ex DC. & & & 1 & & 1 \\
\hline Azorella monantha Clos. & & & 1 & & 1 \\
\hline Desfontainia fulgens D. Don & & & 1 & & 1 \\
\hline Drapetes muscosus Lam. & & & 1 & & 1 \\
\hline Poa denudata Steud. & & & 1 & & 1 \\
\hline Senecio smithii DC. & & & 1 & & 1 \\
\hline Tepualia stipularis (Hook. et Arn.) Griseb. & & & 1 & & 1 \\
\hline Uncinia tenuis Poepp. ex Kunth & & & 1 & & 1 \\
\hline Acaena magellanica (Lam.) Vahl. & & & & 1 & 1 \\
\hline Baccharis magellanica (Lam.) Pers. & & & & 1 & 1 \\
\hline Escallonia alpina Poepp. ex DC. & & & & 1 & 1 \\
\hline Nothofagus pumilio (Poepp. et Endl.) Krasser & & & & 1 & 1 \\
\hline Ranunculus trullifolius Hook. f. & & & & 1 & 1 \\
\hline Griselinia ruscifolia (Gay) Ball. & & & & 1 & 1 \\
\hline Solenomelus segethii (Phil.) Kuntze & & & & 1 & 1 \\
\hline Valeriana lapathifolia Vahl. & & & & 1 & 1 \\
\hline Especies por asociación & 64 & 79 & 71 & 55 & \\
\hline
\end{tabular}

turbera esfagnosa de Lepidothamnus, cuyo histograma de frecuencia indica una menor homogeneidad de los censos (figura 3).

Similitud florística. Presentes en las cuatro comunidades hay $39(36,79 \%)$ especies en común, en tres $14(13,21 \%)$, en dos $18(16,98 \%)$ y exclusivas de una sola comunidad existen 35 (33,02\%) especies; es decir, hay más especies comunes a todas las comunidades que exclusivas de cada una. Entre ellas hay alta afinidad florística y sus diferencias son menores que lo esperado, incluso la turbera pulvinada de Donatia carece de especies exclusivas de ella, encontrándose todas en alguna de las otras comunidades turbosas (cuadro 3). Esta tabla demuestra además que las especies más importantes se presentan en varias comunidades con diferente cobertura promedio. 
Utilizando esta tabla con la cobertura promedio de las especies de cada comunidad se calcularon índices de similitud que demuestran que entre las cuatro comunidades diferenciadas existe una alta afinidad florística, que es ma- yor al considerar la cobertura (cuadro 4). La figura 4 muestra la constelación de especies que grafica los resultados expuestos, indicando que al utilizar el índice de Jaccard, la mayor distancia florística corresponde a la turbera es-
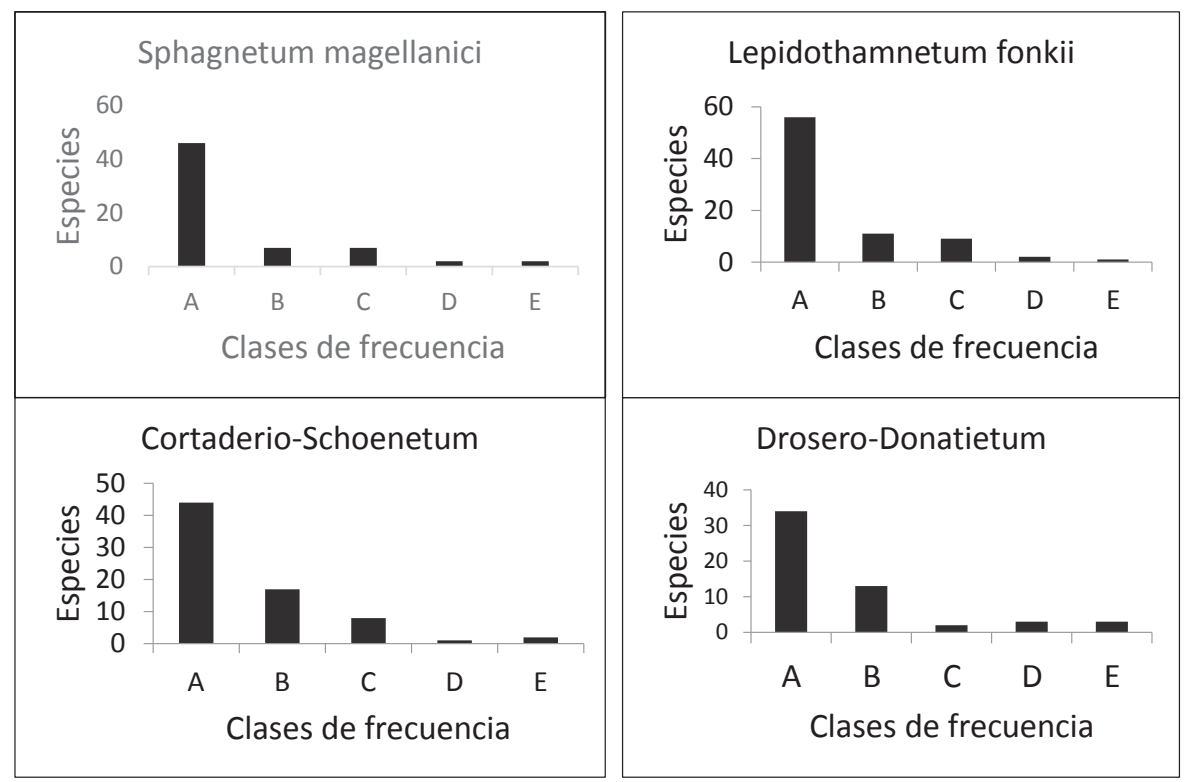

Figura 3. Histogramas de frecuencia de la flora en las comunidades vegetales descritas. Clases de frecuencia: $A=1-20 \%, B=21-40 \%$, $\mathrm{C}=41-60 \%, \mathrm{D}=61-80 \%, \mathrm{D}=81-100 \%$.

Frequency histograms of the described plant communities flora. Frequency: $A=1-20 \%, B=21-40 \%, C=41-60 \%$, $\mathrm{D}=61-80 \%, \mathrm{D}=81-100 \%$.

Cuadro 4. Similitud (triángulos superiores) y disimilitud (triángulos inferiores) entre las distintas comunidades turbosas estudiadas en Aisén (Chile) utilizando el índice de similitud de Jaccard (tabla superior) y de comunidad de Ellenberg (tabla inferior). Abreviaturas de las comunidades: $\mathrm{Sm}=$ Sphagnum magellanicum, $\mathrm{Lf}=$ Lepidothamnus fonkii, $\mathrm{C}-\mathrm{Sa}=$ Cortaderia - Schoenus, $\mathrm{D}-\mathrm{Df}=$ Drosera Donatia fascicularis.

Similarity (upper triangles) and dissimilarity (lower triangles) between the different peat bog studied communities in Aisén (Chile) using the Jaccard similarity index (table above) and Ellenberg community index (table below). Abbreviations of communities; Sm = Sphagnum magellanicum, $\mathrm{Lf}=$ Lepidothamnus fonkii, $\mathrm{C}-\mathrm{Sa}=$ Cortaderia - Schoenus, $\mathrm{D}-\mathrm{Df}=$ Drosera - Donatia fascicularis .

\begin{tabular}{lcccc}
\hline Comunidades & Sm & Lf & C-Sa & D-Df \\
\hline \multicolumn{1}{c}{ Índice de Jaccard } & & & & \\
Comunidad de Sphagnum magellanicum & - & 46,88 & 48,27 & 56.58 \\
Comunidad de Lepidothamnus fonkii & 53,12 & - & 63,04 & 63,41 \\
Comunidad de Cortaderia - Schoenus & 51,73 & 36,96 & - & 63,64 \\
Comunidad de Donatia fascicularis & 43,42 & 36,59 & 36,36 & - \\
\hline \multicolumn{1}{c}{ Índice de Ellenberg } & & & & \\
Comunidad de Sphagnum magellanicum & - & 76,16 & 76,62 & 82,59 \\
Comunidad de Lepidothamnus fonkii & 23,84 & - & 81,77 & 83,91 \\
Comunidad de Cortaderia - Schoenus & 23,38 & 18,23 & - & 82,86 \\
Comunidad de Donatia fascicularis & 14,41 & 16,09 & 17,14 & - \\
\hline
\end{tabular}


fagnosa de Sphagnum magellanicum, en relación con las otras tres comunidades que aparecen más próximas entre sí. Sin embargo la turbera esfagnosa de Lepidothmnus fonkii es florísticamente más cercana a la turbera esfagnosa

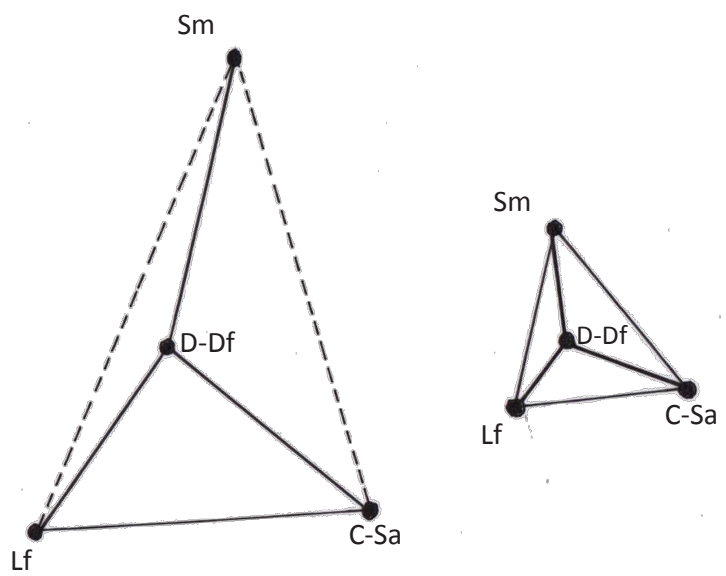

Figura 4. Constelación de comunidades (puntos negros) utilizando la similitud de Jaccard. La figura debe pensarse espacialmente y mientras más gruesas sean las líneas que unen los puntos negros, existe mayor similitud. La menor similitud se indica con línea punteada. Abreviaturas de las comunidades: $\mathrm{Sm}=$ Sphagnum magellanicum, D-Df = Donatia fascicularis. $\mathrm{Lf}=$ Lepidothamnus fonkii, $\mathrm{C}-\mathrm{Sa}=$ Cortaderia- Schoenus . Considerando presencia (izquierda) y cobertura (derecha).

Communities constellation (black dots) using the similarity of Jaccard. The figure should be spatially thought, and the thicker the lines connecting de black dots, the higher the similarity. The least similarities are indicated by a dotted line. Abbreviations of communities: Sm = Sphagnum magellanicum, D-Df = Donatia fascicularis. $\mathrm{Lf}=$ Lepidothamnus fonkii, C-Sa = Cortaderia-Schoenus. Considering presence (left) and coverage (right). de Sphagnum que las otras dos. El índice de Ellenberg muestra una constelación similar, pero de distancias más reducidas entre las comunidades turbosas.

Análisis de conglomerado. El análisis de conglomerados entregó cuatro grupos que se corresponden con las comunidades vegetales diferenciadas por métodos tradicionales (figura 5). Se aprecia una mayor afinidad entre la turbera graminosa de Cortaderia y Schoenus y la turbera pulvinada de Donatia, ambas de mayores altitudes, mientras que la turbera esfagnosa de Lepidothamnus presenta mayor afinidad florística con las dos recién citadas que con la turbera esfagnosa de Sphagnum que aparece como el grupo más aislado.

Análisis de ordenación. La ordenación de los censos muestra a los de la turbera esfagnosa de Sphagnum magellanicum en el extremo izquierdo del primer eje horizontal del análisis NMMS, en el otro extremo se ubican los censos de la turbera pulvinada de Donatia (figura 6). Los de la turbera graminosa de Cortaderia-Schoenus andinus y de la turbera esfagnosa de Lepidothamnus fonkii se sitúan más cerca de la parte media del eje, aunque los primeros corridos hacia la izquierda y los segundos, hacia la derecha. También se presentan agrupados los censos de la turbera esfagnosa y de la turbera graminosa de Cortaderia-Schoenus y relativamente aislados de los otros, lo que indica una mayor afinidad entre ellos, y aquellos de la turbera esfagnosa de Lepidothamnus y pulvinada de Donatia se entremezclan con una mayor dispersión señalando mayor afinidad entre ellos. Los censos de la última comunidad se aproximan a las otras, confirmando el hecho de que ella se instala en partes más altas y secas. Considerando que la turbera esfagnosa de Sphagnum tiene proximidad

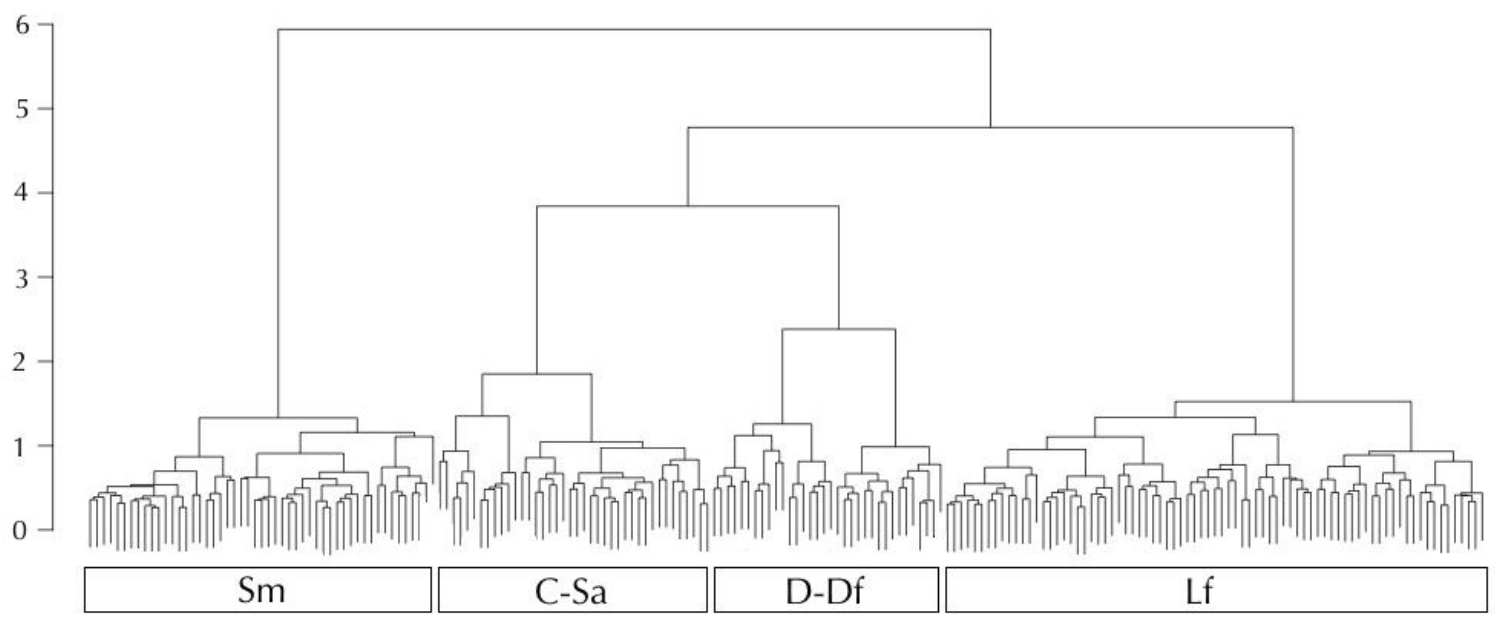

Figura 5. Dendrograma con los 204 censos presentes en la tabla inicial. Abreviaturas de las comunidades: Sm = Sphagnum magellanicum, C-Sa = Cortaderia - Schoenus andinus, D-Df = Donatia fascicularis, Lf = Lepidothamnus fonkii.

Dendrogram showing the 204 vegetation samples of the initial table. Abbreviations of the communities: Sm = Sphagnum magellanicum, C-Sa $=$ Cortaderia - Schoenus andinus,, $\mathrm{D}-\mathrm{Df}=$ Donatia fascicularis, $\mathrm{Lf}=$ Lepidothamnus fonkii. 


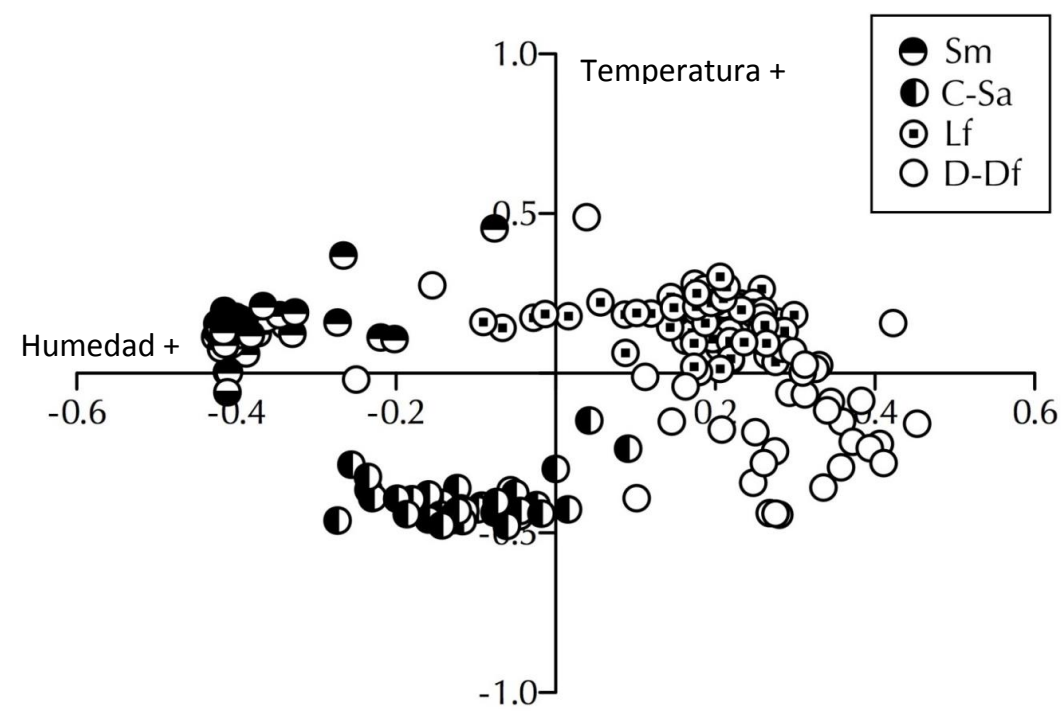

Figura 6. Distribución de los censos de las comunidades vegetales turbosas descritas en los dos primeros ejes del análisis NMMS. Significado de las abreviaturas: Sm = comunidad de Sphagnum magellanicum, C-Sa = Comunidad de Cortaderia-Schoenus andinus, Lf = Comunidad de Lepidothamnus fonkii, D-Df = Comunidad de Drosera-Donatia fascicularis.

Distribution of the vegetation samples of the described peat bog communities in the first two axes of the NMDS analysis. Meaning of the abbreviations: $\mathrm{Sm}=$ community of Sphagnum magellanicum, C-Sa $=$ Community of Cortaderia-Schoenus andinus, Lf $=\mathrm{Community} \mathrm{of} \mathrm{Lepidotham-}$ nus fonkii, D-Df = Community of Drosera-Donatia fascicularis.

del nivel de saturación en sectores y la turbera pulvinada de Donatia, es más seca, se supone que este eje representa un gradiente de humedad del sustrato que incrementa de derecha a izquierda.

El segundo eje vertical presenta casi la misma separación que los otros, si se consideran las distintas escalas de ellos. Este eje separa en el extremo inferior los censos de la turbera graminosa de Cortaderia y Schoenus y de la turbera pulvinada de Donatia y el resto, en el extremo superior, pero más cerca del eje horizontal, lo que indica una escasa diferenciación con respecto al factor representado por este eje, que al parecer se aproxima a un gradiente de temperatura que aumentaría de abajo hacia arriba, lo que se corresponde con la altitud, ya que estas dos comunidades ocupan hábitats de mayores alturas y por consiguiente, más fríos y ventosos.

$\mathrm{Al}$ distribuir las principales especies en el plano formado por ambos ejes, Sphagnum magellanicum, S. fimbriatum y Empetrum rubrum se ubican en el lado izquierdo del primer eje indicando su mayor afinidad hacia una mayor disponibilidad de agua (figura 7). En el otro extremo del mismo eje se ubican Bolax caespitosa, Astelia pumila, Tribeles australis y, especialmente, Donatia fascicularis que prefieren biotopos sin anegamiento y más propensos a secarse en la época estival. De acuerdo al análisis estadístico multivariado de ordenación, estas siete especies serían indicadoras del grado de anegamiento del sustrato, presentando las tres primeras una alta afinidad y las otras cuatro, baja afinidad hacia él. La distribución de las especies en el segundo eje, que correspondería a un gradiente de temperatura, no presenta especies en el extremo inferior $y$, la mayoría de ellas, se ubican en el extremo superior sobre el eje horizontal, es decir, en el lado más frío que correspondería a tierras altas. Entre estas especies destacan Cortaderia egmontiana. Poa bulbosa, Schoenus andinus y Acaena magellanica, todas ellas de turberas de altura. Donatia fascicularis ocupa la posición intermedia, indicando con ello que el factor temperatura no tiene mucha influencia sobre ella, pudiendo encontrarse en tierras altas o bajas con el mismo macroclima.

Las turberas esfagnosas con dominancia de Sphagnum magellanicum que, son las más abundantes, se presentan en bajas altitudes a orillas de cuerpos de agua (lóticos o lénticos), las esfagnosas con dominancia de Lepidothamnus fonkii colonizan planicies anegadas en terrazas de río a una altitud mayor, pero con diferencia de menos de 100 $\mathrm{m}$ de altitud con la primera y no tienen contacto directo con cuerpos de agua, las pulvinadas crecen a mayores altitudes, en laderas y se alimentan del agua de lluvia que retienen a pesar de la inclinación del sustrato que colonizan y las graminosas se encuentran en mayores altitudes, coronando la cumbre de los cerros.

Las turberas son formaciones vegetales primarias (originales) de Chile, no obstante la turbera de Sphagnum magellanicum presenta también estadios secundarios en lugares donde originalmente crecía el bosque de ciprés de Las Guaitecas, el cual fue explotado, quemado, cortado o drenado provocando su muerte. Estas turberas secundarias son abundantes y presentan mayor cobertura de Sphagnum. El resto de las comunidades turbosas encontradas son 


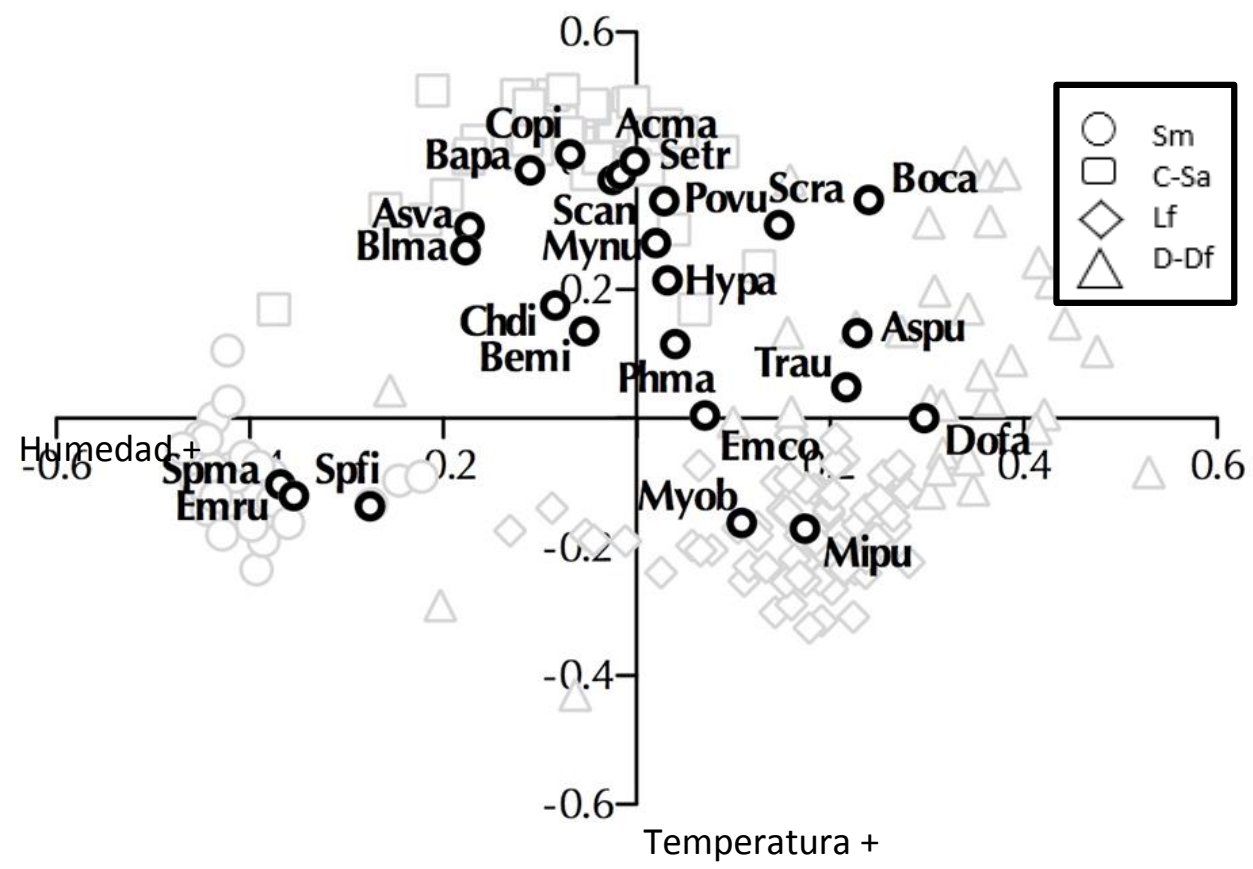

Figura 7. Distribución de las principales especies vegetales de las comunidades turbosas estudiadas (círculos negros). Abreviaturas de arriba hacia abajo: Copi $=$ Cortaderia egmontiana, $\mathrm{Acma}=$ Acaena magellanica, Bapa $=$ Baccharis patagonica, Setr $=$ Senecio triodon, Asva $=$ Symphyotrichum vahlii. Scan $=$ Schoenus andinus, Sera $=$ Azorella ranunculus, Boca $=$ Bolax caespitosa, Blma $=$ Blechnum magellanicum, Mynu= Myrteola nummularia, Hypa = Hypochaeris palustris, Chdi $=$ Chiliotrichum diffusum, Bemi $=$ Berberis microphylla, $\mathrm{Aspu}=$ Astelia pumila, Trau $=$ Tribeles australis, Phma $=$ Philesia magellanica, Emco $=$ Embothrium coccineum, Dofa $=$ Donatia fascicularis, Spma = Sphagnum magellanicum, Emru =Empetrum rubrum, Myob $=$ Myschilos obonga, Mipu $=$ Misodendron punctulatum. Los signos esbozados atrás de los círculos negros señalan la ordenación de los censos de las diferentes comunidades vegetales turbosas descritas (ver figura 6).

Distribution of the main plant species in the studied peat bogs communities (Black circles). Abbreviations from top to down: See Spanish legend. Signs behind the black circles indicate the ordination of vegetation samples of the different described peat bog plant communities (see figure 6).

únicamente primarias y prosperan en Chile desde Valdivia al Sur, siendo componentes importantes de la llamada tundra magallánica por Schmithüsen (1956).

\section{DISCUSIÓN}

En la provincia Capitán Prat de la Región de Aisén se presentan cuatro asociaciones vegetales de turbera: Gaultherio-Sphagnetum magellanici, Schoeno andini-Lepidothamnetum fonkii, Drosero uniflorae-Donatietum fascicularis y Cortadero egmontianae-Schoenetum andinus de ellas, las tres primeras han sido descritas en la literatura (Amigo et al. 2017), mientras que la última se propone como una asociación vegetal nueva (asoc. nova) en este trabajo. Ellas se pueden agrupar en tres tipos: esfagnosas (con dominancia del musgo Sphagnum magellanicum) las dos primeras, pulvinada con una carpeta continua de plantas acojinadas del grupo Angiospermas (plantas con flores) y el tercer grupo, la última, graminosa (muy estratificada y con dominancia de pastos en "champa"), esta última podría considerarse como una situación intermedia entre los dos primeros grupos (Kleinebecker et al. 2010).
Datos recientes para las turberas de Aisén muestran un alto nivel de biodiversidad vegetal en las turberas graminosas, que es claramente menor en las dos turberas esfagnosas, ocupando las pulvinadas una posición intermedia (Sandoval et al. 2016). La similitud florística entre ellas es alta, como lo demuestra el hecho de que existe mayor cantidad de especies comunes a las cuatro asociaciones que aquellas que las diferencian (cuadro 1). La turbera pulvinada carece de especies propias, sin embargo, la abundancia relativa de las que presenta la hacen diferente. Lo anterior también se confirma al compararlas con índices de similitud cuyos valores son altos, a pesar de que presentan diferencias entre ellas. De hecho, la más aislada y con mayor disimilitud con las otras fue la turbera esfagnosa de Sphagnum magellanicum, aun cuando la turbera esfagnosa de Lepidothamnus fonkii se acerca más a ella que las turberas pulvinada y graminosa. Las diferencias encontradas entre las turberas investigadas son mayores en su fisonomía que en su flora.

De acuerdo a los resultados se cumpliría la hipótesis de que la humedad y la temperatura son los factores más importantes en la distribución principalmente altitudinal 
de las turberas en la provincia Capitán Prat de la Región de Aisén. Aunque los análisis multivariados no indican la importancia del fuerte viento, muy frecuente en la Región de Aysén, habría que considerarlo, porque la superficie de las turberas está muy expuesta sin protección arbórea y dependiendo de la exposición esto debe aumentar la evapotranspiración especialmente durante el período estival. Últimamente, las turberas están presentando quemaduras por golpes de sol, como se observa en Oreobolus obtusangulus y en la abundante flora liquénica de ellas (Villagra et al. 2009).

Las turberas, especialmente las de Sphagnum magellanicum están seriamente amenazadas por la explotación indiscriminada del musgo "pon-pon" de Valdivia al Sur y, por la posible extracción de turba para combustible doméstico, en Magallanes (Castillo 2009). Por las razones anteriores, en el año 2018 (Diario Oficial de la República de Chile) con fecha 2017 se publicó un decreto del gobierno chileno que regula su explotación de la parte superior viva del Sphagnum (comúnmente "fibra"), el que fue retirado en posterioridad. Las otras comunidades turbosas de la tundra magallánica, también presentan problemas de conservación, aunque en menor escala. Aunque no tienen utilidad práctica directa, por desconocimiento, sus rodales son considerados secundarios, pensando que reemplazan bosques que fueron explotados. Por ello, en los terrenos ocupados por turberas se permite el establecimiento de nuevas plantaciones y construcciones industriales, cuyos caminos de acceso se transforman en eficientes drenajes que ponen en peligro su existencia con lo que, además, se pierde su rol como reservorios de endemismos y de agua, esta última muy importante en la actualidad principalmente en las cordilleras de Chiloé (Ramírez et al. 2014b). El ganado no se introduce a las turberas, por lo blando del sustrato, pero a veces se aventuran en busca de agua con consecuencias fatales. Desde el punto de vista de la biodiversidad que albergan estos humedales, aunque solo Lepidothamnus fonkii es endémica de Chile, 93 son endémicas de Chile y Argentina en la región patagónica y 6 se comparten con otros países, es decir 100 de las 103 especies de plantas vasculares presentes en las turberas son nativas de Chile y solo tres (Festuca rubra, Poa bulbosa y Juncus planifolius) son introducidas y crecen con coberturas inferiores al $1 \%$. Esto recalca el carácter pristino de las turberas que al parecer incluso fueron respetadas por los incendios provocados en Aisén en el siglo pasado.

Las turberas descritas para la provincia de Capitán Prat, con excepción del Cortaderio egmontianae-Schoenetum andinus se presentan en menor cantidad en la región Norte de Aisén. De acuerdo a nuestras observaciones en este catastro faltaría incluir una comunidad de Astelia pumila y Marsippospermum grandiflorum que se presentaría sobre el límite del bosque, de ella se obtuvieron algunos censos, pero no los suficientes como para definir una comunidad. Estas altitudes son difíciles de alcanzar sin la ayuda de helicóptero. De acuerdo a Álvarez et al. 2010, que estu- diaron cuatro islas (Guamblin, Kent, Stokes e Ipún) del Archipiélago de Los Chonos, en las islas expuestas al Pacífico se presentarían las mismas comunidades de la turbera Gaultherio-Sphagnetum magellanicii y el Drosero uniflori-Donatietum fascicularis. Lo mismo encontraron Ramírez et al. 2014a, en el canal Walker más Sur. Por lo anterior, aún queda territorio por relevar en la Región de Aisén en busca de humedales turbosos.

Esquema sintaxonómico de las asociaciones de turberas de la Región de Aisén descritas en este trabajo, basado en las proposiciones de Amigo et al. (2017):

Clase: Myrteolo nummulariae-Sphagnetea magellanici Oberdorfer 1960

Orden: Myrteolo-Sphagnetalia magellanici Oberdorfer 1960

Alianza: Astelio-Oreobolion obtusanguli Oberdorfer 1960

Asociación: Drosero uniflorae-Donatietum fascicularis Ramírez 1968

Asociación: Schoeno andini-Lepidothamnetum fonkii Ramírez et al. 2014

Alianza: Gaultherio-Sphagnion magellanici Oberdorfer 1960

Asociación: Gaultherio-Sphagnetum magellanici Oberdorfer 1960

Alianza: Cortaderio-Schoenion andinus All. nova

Asociación: Cortaderio egmontianae-Schoenetum andinus ass. nova

\section{CONCLUSIONES}

Las turberas descritas para la provincia de Capitán Prat de la región de Aisén se pueden agrupar en cuatro asociaciones vegetales: Gaultherio-Sphagnetum magellanici, Schoeno andini-Lepidothamnus fonkii, Cortaderio egmotnianae-Schoenetum andinus y Drosero uniflorae-Donatietum fascicularis. Las dos primeras corresponden a turberas esfagnosas de las cuales, una es cosmopolita y la otra propia de Chile. La tercera es una turbera graminosa y la última, es pulvinada. Estas dos últimas están circunscritas al territorio chileno y son avanzadas septentrionales de la zona vegetacional de tundra magallánica chilena, ubicada en las islas expuestas al Pacífico, principalmente al Sur del Estrecho de Magallanes. Aunque las cuatro asociaciones nombradas son fáciles - y fisonómicamente distinguibles, presentan una alta similitud florística. De acuerdo al análisis de ordenación, la humedad del sustrato y la temperatura del aire serían determinantes en la distribución de ellas, en tierras bajas e intermedias las esfagnosas sin y con ciprés enano, respectivamente, y en tierras altas las turberas graminosas y pulvinadas. En este trabajo la turbera graminosa Cortaderio pilosae-Schoenetum andinus se propone como asociación vegetal turbosa nueva para la ciencia. Las turberas chilenas, incluidas las de Sphagnum, son importantes reservorios de la biodiversidad endémica de la región patagónica. 


\section{AGRADECIMIENTOS}

Investigación realizada en el marco del Proyecto CA 012.05 UACh-HYDROAISEN que está dedicada a la memoria del Prof. Dr. Victor Sandoval del Instituto de Bosques y Sociedad de la Facultad de Ciencias Forestales y Recursos Naturales de la Universidad Austral de Chile, quien fuera director del Proyecto.

\section{REFERENCIAS}

Álvarez M, C San Martín, C Novoa, G Toledo, C Ramírez. 2010. Diversidad florística, vegetacional y de hábitats en el Archipiélago de Los Chonos (Región de Aisén, Chile). Anales del Instituto de la Patagonia 38 (1): 34-55. DOI: https://doi. org/10.4067/s0718-686x2010000100002

Álvarez M, BM Möseler, C San Martín. 2013. Tendency and distribution indices for ordinal variables applied to Ellenberg indicator values. Gayana Botánica 70 (2): 225-233. DOI: http://dx.doi.org/10.4067/S0717-66432013000200006

Álvarez M, C San Martín, C Novoa, G Toledo, C Ramírez. 2010. Diversidad florística, vegetacional y de hábitats en el Archipiélago de Los Chonos (Región de Aisén, Chile). Anales del Instituto de la Patagonia 38 (1): 34-55.

Amigo J, C San Martín, C Ramírez, M Álvarez. 2017. Nomenclatural revisión and syntaxonomical proposal for wetland peat vegetation in the Valdivian-Magellanian región. Lazaroa 38 (2): 165-187. DOI: https://doi.org/10.5209/laza.56343

Castillo E. 2009. Factibilidad técnica para la cosecha de Sphagnum magellanicum a microescala. Trabajo de Título para Ingeniero Agropecuario. Punta Arenas, Chile. http://repositorio.conicyt.cl/handle/10533/236363

Clarke KR. 1993. A method of linking multivariate community structure to environmental variables. Marine Ecology Progress Series 92: 205-219. DOI: https://doi.org/10.3354/ meps092205

Dengler J, M Chytry, J Ewald. 2008. Phytosociology. In Jøgensen SE, BD Fath eds. Encyclopedia of Ecology. Oxford, England. Elsevier. p. 2767-2779 DOI: https://doi. org/10.1016/b978-008045405-4.00533-4

Frahm JP. 2001. Biologie der Moose. Alemania, Heidelberg Berlin, Spektrum Akademischer Verlag. 354 p.

Kleinebecker T, N Hölzel, A Vogel. 2010. Patterns gradients of diversity in South Patagonian ombrotrophic peat bogs. Austral ecology 35: 1-12. DOI: https://doi.org/10.1111/j.14429993.2009.02003.x

Knapp R. 1984. Considerations on quantitative parameters and qualitative attributes in vegetation analysis and in phytosociological relevés. In Knapp R ed. Sampling methods and taxon analysis in vegetation science. La Haya, Holand. Junk Publishers. p. 77-119. DOI: https://doi.org/10.2307/2996550

Mc Cune BJ, B Grace. 2002. Analysis of ecological communities. MJM Software, Glenden Beach, Oregon, USA.

Mueller-Dombois D, H Ellenberg. 1974. Aims and methods of vegetation ecology. New York USA, Wiley. 547 p.

Oberdorfer E. 1960. Pflanzensoziologische Studien in Chile - Ein Vergleich mit Europa. Flora et vegetatio mundi 2: 1-208.

Ramírez C. 1968. Die vegetation der Moore der Cordillera Pelada, Chile. Bericht der Oberhessisch. Ges. für Natur- und Heilkunde zu Giessen NF. Naturwiss. Abt. 36: 95-101.
Ramírez C, H Figueroa. 1985. Delimitación ecosociológica del bosque valdiviano (Chile) mediante análisis estadísticos multivariados. Studia Oecologica 6: 105 - 124.

Ramírez C, V Sandoval, C San Martín, M Álvarez, Y Pérez, C Novoa. 2012. El paisaje rural antropogénico de Aisén, Chile: Estructura y dinámica de la vegetación. Gayana Botánica 69 (2): 219-231. DOI: https://doi.org/10.4067/s0717$\underline{66432012000200001}$

Ramírez C, I Ortíz, C San Martín, O Vidal, M Álvarez, Y Pérez, JL Solís, I Álvarez. 2014a. Estudio preliminar de la biodiversidad vegetal terrestre en el Estero Walker (Región de Aysén, Chile): utilizando líneas base de proyectos de inversión. Gayana Botánica 71(3): 227-245. DOI: https:// doi.org/10.4067/s0717-66432014000200006

Ramírez C, C San Martín, O Vidal, Y Pérez, J Valenzuela, JL Solís, G Toledo. 2014b. Tundra subantártica en la Isla Grande de Chiloé, Chile: Flora y Vegetación Turbosa de Campañas. Anales Instituto Patagonia 42(2): 17-37. DOI: $\underline{\text { https:// }}$ doi.org/10.4067/s0718-686x2014000200002

Ramírez C, M Álvarez, C San Martín, E Carrasco, O Vidal, G Toledo, Y Pérez, J Amigo, M Veste. 2018. El cambio climático como causa de daños en bosques chilenos. Chloris chilensis Revista chilena de flora y vegetación 21(1): www.chlorischile.cl.

Rodríguez C. 2015. Hydrogeomorphic classification of mire ecosystems within the Baker and Pascua Basins in the Region Aysén, Chilean Patagonia: a tool for their asseessment and monitoring. Tesis Doctorado, Humboldt Universität zu Berlin. http://repositorio.conicyt.cl/handle/10533/236363

Rodríguez R, A Marticorena. 2019. Catálogo de las plantas vasculares de Chile. Concepción, Chile. Editorial Universidad de Concepción. 424 p.

San Martín C, C Ramírez, H Figueroa. 1999. Análisis multivariable de la vegetación de un complejo de turberas en Cordillera pelada (Valdivia, Chile). Lazaroa 20: 95-106.

San Martín C, C Ramírez, M Álvarez. 2004. Estudio de la vegetación de "Mallines" y "Campañas" en la Cordillera Pelada (Valdivia, Chile). Revista Geográfica de Valparaíso 35: 261-273.

San Martín C, Y Pérez, M Álvarez, C Salazar, C Ramírez. 2014a. Diversidad vegetal de lagunas temporales en la estepa patagónica chilena. Anales Instituto Patagonia 41 (2): 109-114. DOI: https://doi.org/10.4067/s0718$\underline{686 \times 2013000200012}$

San Martín C, V Sandoval, M Álvarez, O Vidal, Y Pérez, JL Solís. 2014b. Comparación de etapas de degradación vegetacional con manejo pecuario utilizando valores bioindicadores de Ellenberg en la Patagonia Chilena. Bosque 35(2): 141-154. DOI: https://doi.org/10.4067/s0717$\underline{92002014000200002}$

San Martín C, D Montenegro, D Vidal, I Álvarez, JL Solís, C Ramírez. 2018. Comparación florística y alteración antrópica de tres turberas esfagnosas en la Región de Aisén, Chile: buscando indicadores de perturbación por manejo. Agro Sur 46 (1): 13-27. DOI: https://doi.org/10.4206/ agrosur.2018.v46n1-03

Sandoval V, C Ramírez, C San Martín, O Vidal, M Álvarez, A Marticorena, Y Pérez. 2016. Diversidad vegetal en las cuencas de los ríos Baker y Pascua (Aisén, Patagonia Chilena). Bosque 37(2): 243-253. DOI: https://doi. org/10.4067/s0717-92002016000200003 
Schmithüsen J. 1956. Die räumliche Ordnung der chilenischen vegetation. Bonner Geographische Abhandlungen 17: 1-86.

Vásquez J. 2008. Características anatómicas, propiedades físicoquímicas y capacidad de retención de agua en gametofitos de Sphagnum magellanicum BRID. En un gradiente latitudinal. Tesis, Escuela de Química y Farmacia. Valdivia, Chile. Facultad de Ciencias, Universidad Austral de Chile. 119 p.

Villagra J, D Montenegro, C San Matín, C Ramírez, I Álvarez. 2009. Estudio de la flora liquénica de las turberas de la comuna de Tortel (Región de Aisén, Patagonia Chilena). Anales Instituto Patagonia (Chile) 37(1): 53-62.
Weber HE, JS Moravec, JP Theurillat. 2000. International Code of Phytosociological Nomenclature. $3^{\text {rd }}$. edition. Journal of Vegetation Science 11: 739-768. DOI: https://doi. org/10.2307/3236580

Wikum D, GF Shanholtzer. 1978. Application of the Braun-Blanquet cover-abundance scale for vegetation analysis in land development studies. Environmental Management 2 (4): 323-329. DOI: https://doi.org/10.1007/bf01866672

Zuloaga F, O Morrone, M Belgrano. 2008. Catálogo de las plantas vasculares del cono sur. Missouri Botanical Garden Press. 3348 p.

Recibido: 02/11/20

Aceptado: 30/04/21 